\title{
Direct Measurement of Decoherence for Entanglement between a Photon and Stored Atomic Excitation
}

\author{
H. de Riedmatten, J. Laurat, C. W. Chou, E. W. Schomburg, D. Felinto, and H. J. Kimble \\ Norman Bridge Laboratory of Physics 12-33, California Institute of Technology, Pasadena, California 91125, USA
}

(Received 29 June 2006; published 14 September 2006)

\begin{abstract}
Violations of a Bell inequality are reported for an experiment where one of two entangled qubits is stored in a collective atomic memory for a user-defined time delay. The atomic qubit is found to preserve the violation of a Bell inequality for storage times up to $21 \mu \mathrm{s}, 700$ times longer than the duration of the excitation pulse that creates the entanglement. To address the question of the security of entanglementbased cryptography implemented with this system, an investigation of the Bell violation as a function of the cross correlation between the generated nonclassical fields is reported, with saturation of the violation close to the maximum value allowed by quantum mechanics.
\end{abstract}

Entanglement between light and matter enables nonclassical correlation between flying and stored quantum states, and as such is a critical resource for quantum information science [1]. Among the capabilities enabled by atom-light entanglement are the teleportation of quantum states of light to a quantum memory [2] and the heralded entanglement between remote atomic systems $([3,4]$ and references therein). Generally, light-matter entanglement provides an essential enabling building block for applications such as scalable quantum networks and quantum repeaters over large distances [5,6]. Beyond the pioneering demonstrations of violations of Bell inequalities by photons spontaneously emitted in atomic cascades $[7,8]$, recent experiments have explicitly demonstrated entanglement between the polarization states of single photons and the internal spin states of single trapped atoms $[9,10]$. On the other hand, the seminal work of Duan, Lukin, Cirac, and Zoller (DLCZ) [6,11] spurred intense experimental and theoretical efforts related to the entanglement of single photons and collective excitations in atomic ensembles. Advances on this front include the generation [12,13], storage [14-16], entanglement [4,17], and transfer from matter to light [18-22] of single collective atomic excitations, as well as probabilistic entanglement between internal atomic Zeeman states and photon polarization [23].

The relevance of atom-light entanglement for quantum network applications arises from the fact that the material qubit can be stored and later converted to a photonic qubit, while preserving its coherence. However, in all experiments to date along this line $[9,10,23]$, no direct study was made of the decoherence process in the qubit storage. For experiments with collective atomic excitations, the entanglement was demonstrated for short storage times (e.g., $500 \mathrm{~ns}$ in [17]), comparable to the duration of the excitation pulse. For quantum memory applications, however, it is clearly important that the storage time is much longer than the time needed to address the memory. Longer coherence times for atomic ensembles in the singleexcitation regime have been inferred from the decay of cross-correlation functions for the emitted light [14$16,22,23]$, but without direct measurements of the lifetime for entanglement.

In this Letter, we report the first direct measurement of decoherence for one stored component of a Bell state in an atomic memory. Polarization entanglement is generated in a probabilistic way between a photon and a collective atomic excitation. After a variable storage time $\tau$, the atomic qubit is transferred into a photon, and the polarization correlation with the initial photon is measured as a function of $\tau$. The violation of a Bell inequality is observed for storage times up to $\tau \simeq 21 \mu \mathrm{s}, 700$ times longer than the duration of the initial excitation pulse $(30 \mathrm{~ns})$. In addition, for small $\tau=400 \mathrm{~ns}$, we measure the Bell parameter $S$ as a function of the normalized cross correlation $g_{12}$ between the initial and retrieved photons, thereby addressing operationally the relationship between the nonclassical character of the generated fields and the security of a quantum channel implemented with these resources [24]. Our observations are made possible by two advances, namely, a large improvement of the quality of the photon pairs emitted by the atomic ensemble [21], and the implementation of conditional logic for the generation and readout of the stored qubit.

Figure 1 provides an overview of our experiment, with (a) and (b) illustrating the relevant pathways to generate entanglement probabilistically between a photon and a collective atomic excitation, and (c) showing the experimental setup. The optically thick atomic ensemble is obtained from cold $\mathrm{Cs}$ atoms in a magneto-optical trap (MOT). We call $\{|g\rangle,|s\rangle,|e\rangle\}$ the hyperfine levels $\left\{\left|6 S_{1 / 2}, F=4\right\rangle,\left|6 S_{1 / 2}, F=3\right\rangle,\left|6 P_{3 / 2}, F=4\right\rangle\right\}, \quad$ respectively. With initially all atoms in the ground state $|g\rangle$, a weak write pulse, detuned $10 \mathrm{MHz}$ below the $g \rightarrow e$ transition and right circularly polarized $\left(\sigma^{+}\right)$, passes through the sample. With small probability $p$, an atom in $\left|g, m_{F}\right\rangle$ 

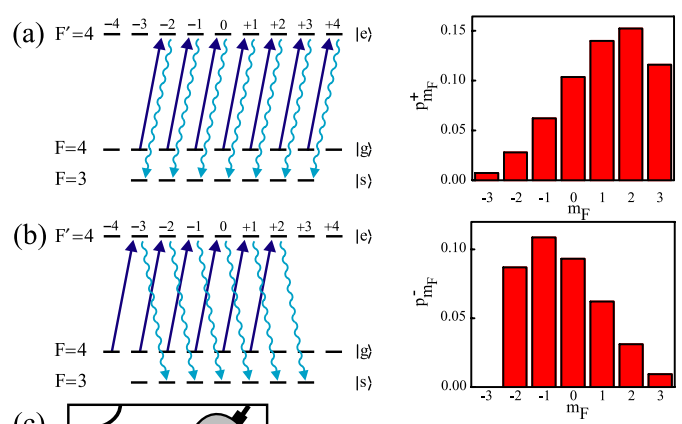

(c)
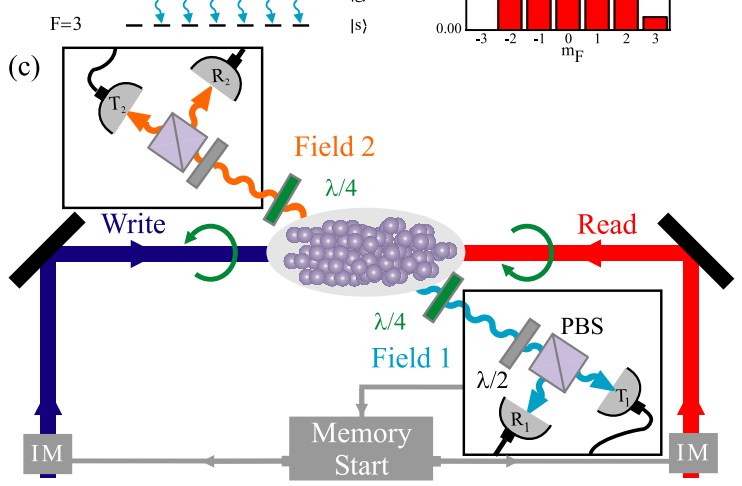

FIG. 1 (color online). (a),(b) Relevant levels and decay paths from $|e\rangle$ to $|s\rangle$, starting from an unpolarized Cs ensemble. The distributions $p_{m_{F}}^{+}, p_{m_{F}}^{-}$for populations in $|s\rangle$ from the two possible decay paths are also shown. (c) Experimental setup. Write and read pulses are sent sequentially with $400 \mathrm{~ns}$ delay, until a detection in field 1 occurs. This event triggers the "memory start" circuit, which stops the write or read sequence for a programmable time $\tau$ by way of independent electro-optic Mach-Zehnder intensity modulators (IM). The read beam power is $\approx 150 \mu \mathrm{W}$, while the write beam power is much weaker $(\mu \mathrm{W}$ range, see text).

undergoes spontaneous Raman scattering by way of the excited state $\left|e, m_{F}^{\prime}=m_{F}+1\right\rangle$ and is thereby transferred to $|s\rangle$ while emitting a photon (field 1). The spatial mode for field 1 is defined by the backwards projection of our imaging system into the ensemble [21]. The transition $\left|g, m_{F}\right\rangle \rightarrow|s\rangle$ via $\left|e, m_{F}^{\prime}=m_{F}+1\right\rangle$ proceeds by two different pathways: by emitting a $\sigma^{+}$polarized photon, arriving at $\left|s, m_{F}\right\rangle$, and by emitting a $\sigma^{-}$photon, arriving then at $\left|s, m_{F}+2\right\rangle$, as in Figs. 1(a) and 1(b), respectively. Also shown are the expected distributions $p_{m_{F}}^{+}, p_{m_{F}}^{-}$of atoms in $|s\rangle$ as a result of $\sigma^{+}, \sigma^{-}$emission assuming an uniform initial distribution among the various $\left|g, m_{F}\right\rangle$. If the relevant emission processes are indistinguishable in all other degrees of freedom, and field 1 is detected in a superposition state of $\sigma^{+}$and $\sigma^{-}$, then the state of the excitation stored in $|s\rangle$ is projected into a coherent superposition of the mixed states shown in Figs. 1(a) and 1(b). In our experiment, the persistency of this projection is evaluated as a function of the storage time $\tau$.

Before detection of the first photon, the joint state of the atom-light system for atoms initially in $\left|g, m_{F}\right\rangle$ can be written as $\rho_{1 a}=|0\rangle\left\langle 0|+| \Phi_{1 a}\right\rangle\left\langle\Phi_{1 a}\right|$, where the nonvacuum part is in the ideal case,

$$
\left|\Phi_{1 a}\right\rangle=\sqrt{p}\left[\cos \eta_{m_{F}}\left|1_{1}^{+}, 1_{a}^{+}\right\rangle+\sin \eta_{m_{F}}\left|1_{1}^{-}, 1_{a}^{-}\right\rangle\right]+O(p),
$$

and $\left|1_{1}^{\alpha}\right\rangle$ represents a photon in field 1 with a polarization $\sigma^{\alpha}$ and $\left|1_{a}^{\alpha}\right\rangle$ the collective atomic states with one excitation as shown in Figs. 1(a) and 1(b), for $\alpha=\{+,-\}$. The parameter $\eta_{m_{F}}$ is obtained from $\cos ^{2} \eta_{m_{F}}=p_{m_{F}}^{+} /\left(p_{m_{F}}^{+}+\right.$ $\left.p_{\left(m_{F}+2\right)}^{-}\right)$. For the more general case where the initial state is an incoherent distribution of the various $\left|g, m_{F}\right\rangle$, the collective atomic states are mixed states and the global $\eta$ is obtained from $\cos ^{2} \eta=\sum p_{m_{F}}^{+} / \sum\left(p_{m_{F}}^{+}+p_{m_{F}}^{-}\right)$[23], where for the case of Cs atoms $\eta=0.86 \times \pi / 4$. Note that the vacuum part in $\rho_{1 a}$ also contains all the light emitted by the ensemble which is not collected in the single mode of our imaging system [11]. By sending a strong read pulse, $\sigma^{-}$polarized with respect to the atoms and resonant with the $s \rightarrow e$ transition, the atomic qubit can be transferred efficiently into a single photon (field 2). Field 2 is emitted into a well-defined spatial mode [21] and with polarization orthogonal to field 1 thanks to a collective enhancement effect $[6,16]$. Hence, the atomic qubit is mapped onto a photonic qubit with, for each $m_{F}$, the atomic state $\left|1_{a}^{\alpha}\right\rangle$ being mapped onto a photonic state $\left|1_{2}^{-\alpha}\right\rangle$ in field 2 .

Returning to the experimental setup in Fig. 1(c), we carry out the excitation and retrieval in a cyclic fashion. At a frequency of $40 \mathrm{~Hz}$, the MOT magnetic field is switched off for $6 \mathrm{~ms}$. After waiting for $3.8 \mathrm{~ms}$ for the magnetic field to decay [16], a sequence of 1100 trials of duration $2 \mu \mathrm{s}$ begins. For each trial, the atoms are initially prepared in $|g\rangle$ with $1 \mu$ s of repumping light. Write and read pulses, each of $30 \mathrm{~ns}$ duration, are mode matched and counterpropagate through the ensemble with a beam waist $\simeq 200 \mu \mathrm{m}$. Fields 1 and 2 are collected with a $3^{\circ}$ angle relative to the write and read beams [19,21,23], and with a waist in the MOT $\simeq 50 \mu \mathrm{m}$. They are then directed to $\lambda / 4$ plates that map circular to linear polarization, and then to rotatable polarizers with angles $\theta_{1}, \theta_{2}$, each consisting of a $\lambda / 2$ plate and a polarization beam splitter (PBS). The two outputs of the PBSs are then coupled to single-mode optical fibers and sent to silicon avalanche photodiodes, denoted by $\left(T_{1,2}, R_{1,2}\right)$ for the transmitted and reflected outputs, for fields 1 and 2, respectively. Before detection, field 1 is sent through a paraffin-coated vapor cell containing Cs atoms in the $|g\rangle$ state, used as a frequency filter to reduce uncorrelated background events [21]. Finally, the detection electronic signals are sent to a data acquisition card, where they are time stamped and recorded for later analysis.

Before studying the storage process, a first characterization at short storage times $\tau=400 \mathrm{~ns}$ is obtained by way of the correlation function $E\left(\theta_{1}, \theta_{2}\right)$ defined by

$$
E\left(\theta_{1}, \theta_{2}\right)=\frac{C_{T_{1} T_{2}}+C_{R_{1} R_{2}}-C_{T_{1} R_{2}}-C_{R_{1} T_{2}}}{C_{T_{1} T_{2}}+C_{R_{1} R_{2}}+C_{T_{1} R_{2}}+C_{R_{1} T_{2}}} .
$$

Here $C_{T_{1} T_{2}}$ gives the number of coincidences between detectors $T_{1}$ and $T_{2}$ for the angles $\theta_{1}$ and $\theta_{2}$. For the generation of photon pairs from an atomic ensemble, the "quality" of the pairs depends on the intensity of the 
excitation (writing) laser [21], as in parametric downconversion. For low excitation intensity, the nonvacuum part is well approximated by a photon pair, but as the excitation increases, the higher order terms can no longer be neglected. We assess the contributions of these higher order terms by way of measurements of the normalized cross-correlation function $g_{12}$ between the two fields, where $g_{12}=p_{12} /\left(p_{1} p_{2}\right)$, with $p_{12}$ as the joint probability for detection events from fields 1 and 2 in a given trial, and $p_{i}$ as the probability for unconditional detections in field $i$. For our system $g_{12}>2$ is a strong indication of a nonclassical state of light for the two fields $[12,16]$.

An example of $E\left(\theta_{1}, \theta_{2}\right)$ is shown in the inset of Fig. 2, where $E$ is displayed as a function of $\theta_{2}$ for two different values of $\theta_{1}=0^{\circ},-45^{\circ}$ corresponding to the projection of photon 1 on bases separated by $45^{\circ}$. These curves were taken for an average value of $\bar{g}_{12}=57$, measured at the point of maximal correlation $\left(\theta_{1}=\theta_{2}=0^{\circ}\right)$, so that the transmitted field 1 (field 2) is $\sigma^{-}\left(\sigma^{+}\right)$polarized. The value $\bar{g}_{12}$ is the average between the two polarization processes described in Figs. 1(a) and 1(b), i.e., where fields 1 and 2 are detected with $\sigma^{+}$and $\sigma^{-}$polarization $\left(\sigma^{+}, \sigma^{-}\right)$, and vice versa $\left(\sigma^{-}, \sigma^{+}\right)$. From measurements of $E\left(\theta_{1}, \theta_{2}\right)$, it is possible to determine the Bell parameter $S=E\left(\theta_{1}, \theta_{2}\right)+$ $E\left(\theta_{1}^{\prime}, \theta_{2}\right)+E\left(\theta_{1}, \theta_{2}^{\prime}\right)-E\left(\theta_{1}^{\prime}, \theta_{2}^{\prime}\right)$ [7]. We use the canonical settings $\theta_{1}=-22.5^{\circ}, \theta_{1}^{\prime}=22.5^{\circ}, \theta_{2}=0^{\circ}, \theta_{2}^{\prime}=45^{\circ}$, which give $S=2 \sqrt{2}$ for an ideal entangled state of two qubits. This corresponds to the maximal violation of the Bell-Clauser-Horne-Shimony-Holt (CHSH) inequality $|S| \leq 2$ [7].

Our results for $S$ for fixed $\tau=400 \mathrm{~ns}$ are displayed in Fig. 2 as a function of $\bar{g}_{12}$ as the write beam power is varied from $\approx 0.3$ to $20 \mu \mathrm{W}$. Violations of the CHSH inequality are evident for large $\bar{g}_{12}$, but are lost as $\bar{g}_{12}$ is reduced. This

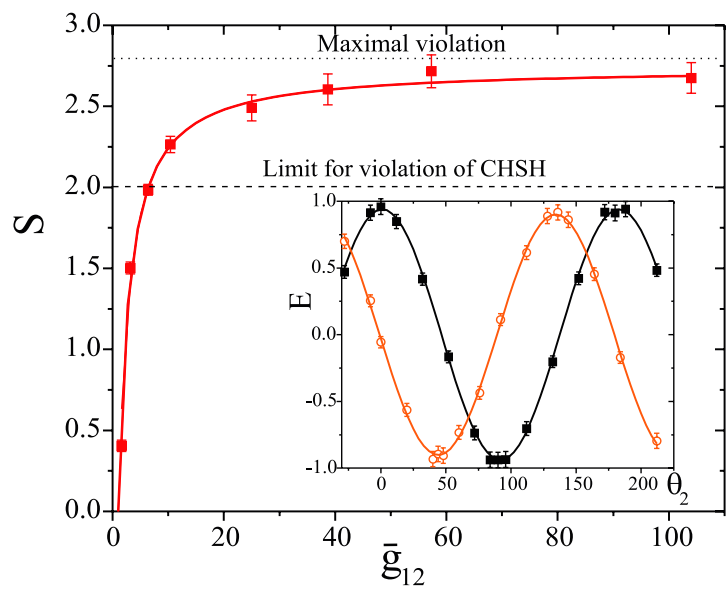

FIG. 2 (color online). Measurement of the Bell parameter $S$ as a function of the average value for the normalized crosscorrelation function $\bar{g}_{12}$. The fitted $S_{\max }$ is $2.74 \pm 0.04$. Inset: Measurement of the correlation function $E\left(\theta_{1}, \theta_{2}\right)$ as a function of $\theta_{2}$, for an average $\bar{g}_{12}=57$. Solid squares shows the fringe for $\theta_{1}=0^{\circ}(V=0.94 \pm 0.02)$, while open circles correspond to $\theta_{1}=-45^{\circ}(V=0.90 \pm 0.02)$. loss is due to the higher order terms, which act as background noise that tends to reduce the visibility $V$ of the fringes in $E\left(\theta_{1}, \theta_{2}\right)$. Since $p_{1} p_{2}$ gives a good estimation for the uncorrelated background, $V$ can be approximated by

$$
V \simeq \frac{p_{12}-p_{1} p_{2}}{p_{12}+p_{1} p_{2}}=\frac{g_{12}-1}{g_{12}+1}
$$

The solid line in Fig. 2 is a fit with the expression $S=$ $S_{\max } V$, where $S_{\max }$ is the maximal possible violation [25], and $V$ is given by Eq. (2). Consistent with Eq. (2), $S$ reaches a plateau for high $\bar{g}_{12}$ with the fitted value $S_{\max }=$ $2.74 \pm 0.04$ close to the maximal violation 2.79 expected for a process with $\eta=0.86 \times \pi / 4$ [23]. Our maximum measured value is $S=2.7 \pm 0.1$, near the maximal violation and representing a violation by 7 standard deviations of the CHSH inequality. Also of note is that the threshold $|S|=2$ for violation of the CHSH inequality occurs for $\bar{g}_{12} \simeq 7$. Although there has been tremendous progress in the achievable value of $g_{12}$ in recent years [21,22], no study has previously investigated the relationship of the quantum correlations represented by $g_{12}$ with the requirements for quantum network applications (e.g., violation of a Bell inequality for the security of entanglement-based quantum cryptography $[24,26])$. The measurements in Fig. 2 represent the first step to quantify this connection.

We next investigate the time interval $\tau$ over which excitation can be stored in the atomic memory while still preserving sufficient coherence for violation of the $\mathrm{CHSH}$ inequality. For this study, the period of the trials must be increased to beyond the decoherence time for the stored qubit (up to $\tau_{\max }=40 \mu \mathrm{s}$ in our case). Because the success probability $p_{1}$ for a detection event from field 1 is necessarily small $\left(p_{1} \simeq 10^{-4}\right.$ for $\left.\bar{g}_{12} \simeq 60\right)$, the time $t_{s}$ required for successful detection becomes long $\left(t_{s} \simeq\right.$ $\left.\tau_{\max } / p_{1}\right)$, leading to prohibitively low count rates if the experiment were to be conducted in the usual cyclic fashion. To circumvent this problem, we have developed a control system that stops all light pulses for a programmable time $\tau$ conditioned upon a detection event for field 1 , before the read pulse is fired. Operationally, the repetition rate for our experiment is thereby increased by more than a factor of 20 as compared to usual (unconditional) cycling.

The open circles in Fig. 3 give the results for our measurement of $S$ as a function of $\tau$. With the same set of angles as in Fig. 2, we find $S=(2.31 \pm 0.17)>2$ at $\tau=20.7 \mu$ s delay. Hence, the storage of the atomic qubit preserves the violation of the CHSH inequality up to $\tau \simeq$ $21 \mu \mathrm{s}$, corresponding to $4 \mathrm{~km}$ propagation delay in an optical fiber. The principal cause for the decay of $S$ with increasing $\tau$ is the residual magnetic field that inhomogeneously broadens the ground state levels $\left|g, m_{F}\right\rangle,\left|s, m_{F}^{\prime}\right\rangle$, as was studied in detail in Ref. [16].

To substantiate this claim, we display in Fig. 3 measurements of $g_{12}$ for the two different polarization configurations $\left(\sigma^{+}, \sigma^{-}\right)$and $\left(\sigma^{-}, \sigma^{+}\right)$taken at the same time as those for $S . g_{12}$ likewise exhibits decay with increasing $\tau$ 


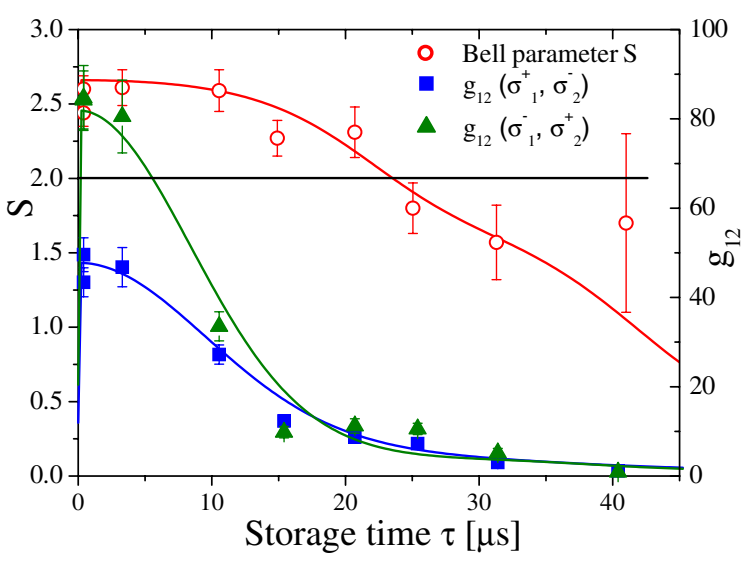

FIG. 3 (color online). Measurement of the Bell parameter $S$ (open circles) and of $g_{12}$ for the two different polarization configurations (solid symbols) as a function of $\tau$. To increase the repetition rate, repumping light has not been used between trials and the period has been shortened to $1.45 \mu \mathrm{s}$, such that 1400 trials can be performed in a measurement period.

that we investigate by applying the model introduced in Ref. [16]. We calculate the joint probability $p_{12}^{\text {th }}(\tau)$ to generate a pair of photons in fields 1 and 2 . We then compare the quantity $p_{12}(\tau)=\xi p_{12}^{\text {th }}(\tau)$ to the measured $g_{12}(\tau)$ by way of a single overall scaling parameter $\xi$ for all $\tau$, for each polarization configuration, resulting in the solid lines in Fig. 3. The observed decay is consistent with an inhomogeneity of the Zeeman splitting across the ensemble described by the parameter $K=\mu_{B} g_{F g} L b / h$, where $L$ is the ensemble length, $b$ the residual magnetic field gradient, and $g_{F g}$ the Landé factor. The fits in Fig. 3 are for $K=12 \mathrm{kHz}$ for the two polarization configurations, which is consistent with the linewidth of the ground states determined independently by stimulated Raman spectroscopy [16]. We have no definitive explanation for the different measured values of $g_{12}$ at $\tau=0$ for the two configurations but suggest that this difference might be due to different backgrounds (consistent with the different $\xi$ values for the two curves). The conditional probability $p_{c}$ to detect a photon in field 2 conditioned on a detection in field 1 follows the decay of $g_{12}$, starting at $6 \%$ for $\tau=0$ and falling to $0.7 \%$ for $\tau=20.7 \mu \mathrm{s}$.

From the theoretical curves for $g_{12}$ in Fig. 3, we obtain a prediction for the decay of the Bell parameter $S$, also shown by a solid curve in Fig. 3. Explicitly, we assume as before that $S=S_{\max } V$ [25], with the visibility $V$ calculated from the average $\bar{g}_{12}$ of the modeled decay for the two polarization configurations by way of Eq. (2) and the $S_{\max }=2.74$ obtained from the fit in Fig. 2. The agreement between this simple model and our measured values of $S$ indicates that the principal cause of decoherence for the Bell-inequality violation is well understood.

In summary, we have described a Bell experiment based on probabilistic entanglement between a photon and a collective atomic excitation, where one of the qubits is stored in an atomic ensemble before being transferred to a single photon. Within the setting of the realization of scalable quantum networks via the protocol of DLCZ [6], we have presented the first measurements to explore the connection between traditional field correlations as expressed by $g_{12}$ and entanglement as represented by the Bell parameter $S$. The storage of the matter qubit leads to a violation of the $\mathrm{CHSH}$ inequality for storage times up to $21 \mu \mathrm{s}$, with the mechanism for decoherence identified theoretically. For scalable quantum networks with a number of nodes $\gg 2$, the coherence time should be much larger than the time needed to create the entanglement with high probability, which remains an experimental challenge [16]. Beyond the setting of the DLCZ protocol, our results represent the first direct measurements of the decoherence in the storage of a matter qubit in an atomlight entanglement experiment.

We gratefully acknowledge our ongoing collaboration with Dr. S. J. van Enk. This work was supported by the DTO of the DNI and by the NSF. J.L. acknowledges financial support from the EU (Marie Curie Fellowship), and D. F. from CNPq (Brazilian agency).

[1] P. Zoller et al., Eur. Phys. J. D 36, 203 (2005).

[2] J. Sherson et al., quant-ph/0605095.

[3] L.-M. Duan et al., Quantum Inf. Comput. 4, 165 (2004).

[4] C. W. Chou et al., Nature (London) 438, 828 (2005).

[5] H.-J. Briegel et al., Phys. Rev. Lett. 81, 5932 (1998).

[6] L.-M. Duan et al., Nature (London) 414, 413 (2001).

[7] J. F. Clauser and A. Shimony, Rep. Prog. Phys. 41, 1881 (1978).

[8] A. Aspect, P. Grangier, and G. Roger, Phys. Rev. Lett. 49, 91 (1982).

[9] B. B. Blinov et al., Nature (London) 428, 153 (2004).

[10] J. Volz et al., Phys. Rev. Lett. 96, 030404 (2006).

[11] L.-M. Duan, J. I. Cirac, and P. Zoller, Phys. Rev. A 66, 023818 (2002).

[12] A. Kuzmich et al., Nature (London) 423, 731 (2003).

[13] M. D. Eisaman et al., Phys. Rev. Lett. 93, 233602 (2004).

[14] T. Chanelière et al., Nature (London) 438, 833 (2005).

[15] M. D. Eisaman et al., Nature (London) 438, 837 (2005).

[16] D. Felinto et al., Phys. Rev. A 72, 053809 (2005).

[17] D. N. Matsukevich et al., Phys. Rev. Lett. 96, 030405 (2006).

[18] C. W. Chou et al., Phys. Rev. Lett. 92, 213601 (2004).

[19] V. Balić et al., Phys. Rev. Lett. 94, 183601 (2005).

[20] A. T. Black, J. K. Thompson, and V. Vuletić, Phys. Rev. Lett. 95, 133601 (2005).

[21] J. Laurat et al., Opt. Express 14, 6912 (2006).

[22] D. N. Matsukevich et al., Phys. Rev. Lett. 97, 013601 (2006).

[23] D. N. Matsukevich et al., Phys. Rev. Lett. 95, 040405 (2005).

[24] A. Ekert, Phys. Rev. Lett. 67, 661 (1991).

[25] I. Marcikic et al., Phys. Rev. Lett. 93, 180502 (2004).

[26] M. Curty et al., Phys. Rev. A 71, 022306 (2005). 